\title{
Accessory coracobrachialis muscle with two bellies and abnormal insertion - case report
}

\author{
George Paraskevas, Konstantinos Koutsouflianiotis, Kalliopi Iliou, \\ Theodosis Bitsis, Panagiotis Kitsoulis
}

Department of Anatomy, Faculty of Medicine, Aristotle University of Thessaloniki, Thessaloniki, Greece

\author{
Correspondence: \\ g_paraskevas@yahoo.gr \\ Tel.: + 302310999330 \\ Fax.: +302310819831
}

Received: 1 June 2016

Accepted: 26 October 2016

Key words: Coracobrachialis muscle Variation - Clinical applications.

\begin{abstract}
Objective. In the current study a brief review is presented of the coracobrachialis muscle's morphological variability, action, embryological development and clinical significance. Case report. We report a case of a left-sided coracobrachialis muscle consisting of two bellies. The deep belly inserts into the usual site in the middle area of the anteromedial aspect of the left humerus, whereas the superficial belly inserts through a muscular slip into the brachial fascia and the medial intermuscular septum, forming a musculo-aponeurotic tunnel in the middle region of the left arm, for the passage of the median nerve, brachial artery and veins, medial antebrachial cutaneous nerve and ulnar nerve. Conclusion. Awareness of such a muscle variant should be kept in mind by physicians and surgeons during interpretation of neural and vascular disorders of the upper limb, since such a variant may potentially lead to entrapment neuropathy and/or vascular compression, predisposing to neurovascular disorders, as well as during preparation of that muscle in cases of utilizing it as a graft in reconstruction of defects.
\end{abstract}

\section{Introduction}

The coracobrachialis muscle $(\mathrm{CBM})$ is a widely known muscle of the anterior compartment of the arm that has received little attention from anatomists and clinicians, due to its minor importance for the forward flexion of the arm. However, the CBM has recently received greater surgical significance since it is utilized as a graft in reconstructive surgery after mastectomy, in the treatment of defects of the axillary and infraclavicular area (1), or in therapy for long standing facial palsy (2). Apart from the CBM's usual insertion site into the median portion of the antero-medial aspect of the humerus, the muscle may also insert into variable anatomical sites, such as the surgical neck of the humerus (3), the tendon of the latissimus dorsi (4), the medial head of the triceps (5), the brachial fascia (6), the medial epicondyle of the humerus or the antebrachial fascia (7).

However, the presence of a variant CBM type, as the finding of the current study, where its superficial portion participates in the formation of a musculo-aponeurotic tunnel including median, ulnar nerves, as well the brachial vessels, has not been detected so frequently. This type of CBM muscular slip could under circumstances such as hypertrophy, hematoma or strong CBM contraction under resistance, predispose to 
median and/or ulnar neuropathy, as well as to vascular disorders.

In the current study a brief review of the CBM's morphological variability, action, embryological development and clinical significance is presented.

\section{Case report}

During routine educational dissection an abnormal CBM was detected in an adult male cadaver, aged 78 years old, on the left side. In particular, after meticulous preparation and

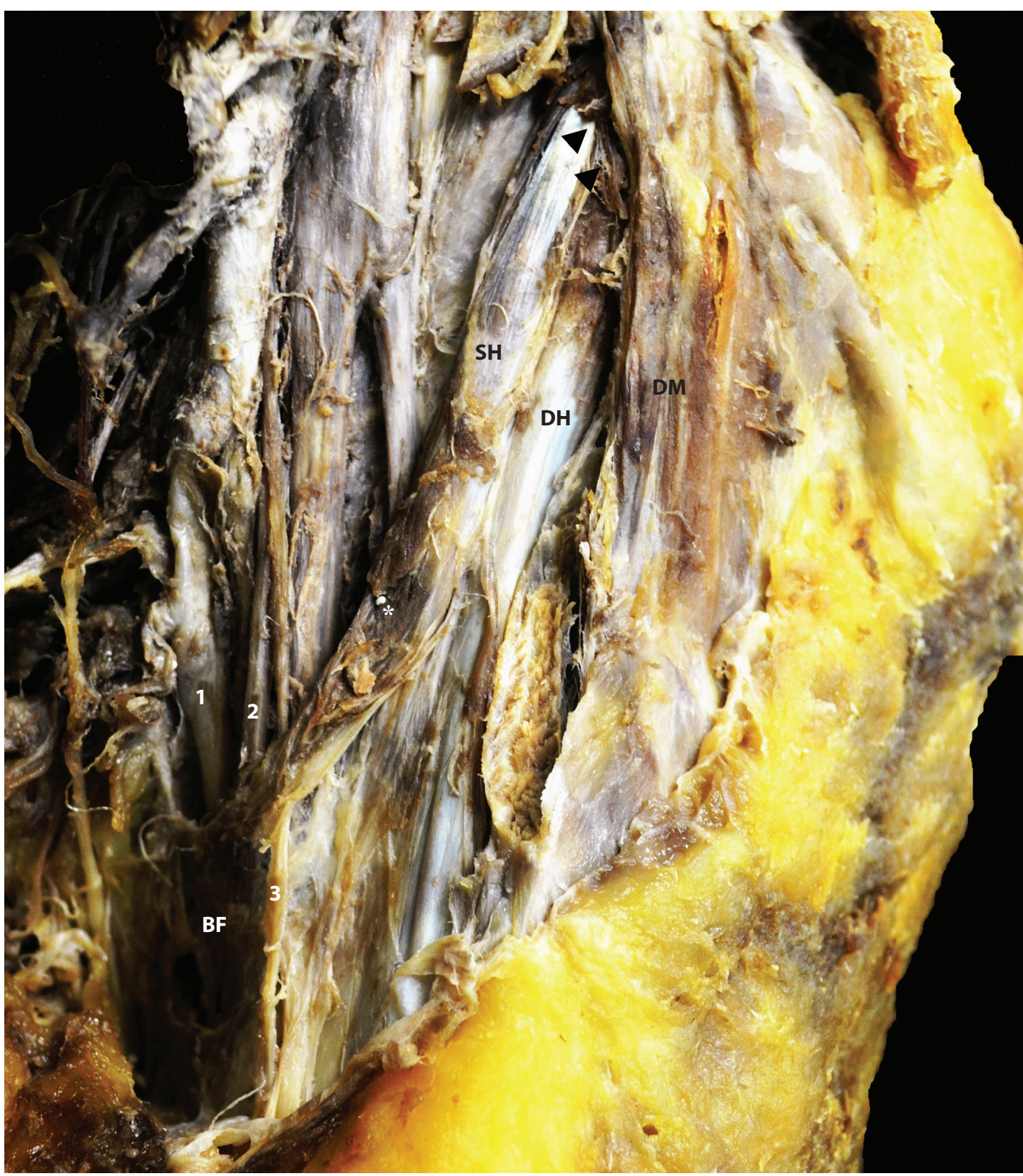

Figure 1 An accessory coracobrachialis muscle on the anterior aspect of the left arm is shown, composed of a superficial head (SH) and deep head (DH). The SH terminates into a muscular slip (asterisk) that inserts into the brachial fascia (BF). The musculo-aponeurotic channel formed in that way contains the nerves and vessels of the medial bicipital groove of the arm (1: brachial artery, 2: median nerve, 3: medial antebrachial cutaneous nerve, DM: deltoid muscle). 
dissection of the skin and the underlying fascia of the left arm region, we came across a bicipital CBM, consisting of a superficial and a deep belly originating together from the tip of the coracoid process. The deep belly terminated via a musculo-aponeurotic tendon into its usual insertion site, that is the middle area of the antero-medial aspect of the left humerus. The superficial belly terminated through a muscular slip into the brachial fascia and the medial intermuscular septum. The latter muscular slip formed a musculo-aponeurotic channel in the middle region of the left arm for the passage of the median nerve, brachial artery and veins, the medial antebrachial cutaneous nerve and the ulnar nerve (Figure 1). It should be noted that the $\mathrm{CMB}$ on the right side did not display any morphological variations. The cadaver was fixed by formalin and alcohol solution. The cause of death was unrelated to the current study, whereas no other variations, pathological conditions or evidence of previous surgical procedures were present in the arm. The morphology and topographic relationship of the current variant were recorded with repeated photographs.

\section{Discussion}

As is widely known, the CBM originates from the apex of the coracoid process, along with the tendon of the short head of the biceps, and by muscular fibers from the proximal part of that tendon. The CBM terminates in an impression, midway across the medial border of the humeral shaft (8). However, the CBM's origin and its insertion display great variability. As regards the CBM's insertion, which is the case in the current study, it may be located in various anatomical sites, from the shoulder joint capsule to the medial epicondyle, the olecranon process or the antebrachial fascia $(7,9,10)$.

As long ago as in 1867, John Wood described three portions of the CBM. The up- per or short portion is the smallest, originating from the coracoid process and inserted into the shoulder joint capsule. This portion was termed by Wood the "coracobrachialis superior or brevis or rotator humeri". The lower or long portion is inserted into the internal condyloid ridge, the internal intermuscular septum or the trochlea, and was termed by Wood the "coracobrachialis longus". The middle portion of the muscle is the largest and is inserted into the middle of the inner surface of the humerus. This portion of the CBM was termed the "coracobrachialis proprius or medius" by Wood (9). Wood considered that the middle portion is usually found in human subjects (9), however other authors speculated that the middle and lower portions are fused, trapping the musculocutaneous nerve between them (4, 6). However, there are instances (3.5-6.5\%) in which the CBM is not traversed by the musculocutaneous nerve (4). Mori observed in $6 \%$ of cases that the course of the musculocutaneous nerve is on the ventral surface of the CBM (11).

Apart from Wood's classification system of the CBM's morphology and attachment sites, in 1964 Mori mentioned the existence of the CBM's separation into superficial and deep layers. In particular, in $16 \%$ of cases the CBM's belly was completely separated, whereas in $8 \%$ it was incompletely separated into a superficial and a deep layer (11). In our case, the CBM displays two muscular heads, one superficial and one deep. The deep head is inserted into the medial aspect of the humerus as usual, whereas the superficial head terminates through a muscular slip into an aponeurotic lamina, which blends into the brachial fascia and the medial intermuscular septum. The latter head of the CBM creates a fibro-muscular tunnel for the passage of the vessels and nerves of the medial bicipital groove of the arm. This variant resembles that mentioned by Ray et al. where two CBM bellies were displayed, 
with the superficial one inserted into the brachial fascia (6). Our case differs from this in that the deep CBM head is musculo-aponeurotic, whereas the deep belly in Ray et al. was totally muscular. Furthermore, the current finding resembles the abnormal muscle observed by Paraskevas et al. that originated from the CBM and the tendon of the long head of the biceps brachii, and inserted into the medial intermuscular septum and the brachial fascia, forming a musculo-aponeurotic channel for the passage of the nerves and vessels of the arm (12). We suggest that our abnormal muscle consists of a superficial layer of the CBM corresponding to the CBM's lower portion.

Some cases of additional CBM heads have been reported in the relevant literature. Chouke noted an accessory CBM head arising from the conoid ligament of the clavicle, blending with the main $\mathrm{CBM}$ and inserting into the medial intermuscular septum (13). Previously, Wood noticed an accessory head inserted into the internal condyloid ridge of the humerus, as well as additional head inserted into the fibrous capsule of the shoulder joint (9). Gupta et al. observed an additional CBM head, which originated from an abnormal site on the coracoid process and inserted via a long thin aponeurotic tendon into the CBM's usual insertion site (14). An accessory CBM was noted by Kopuz et al., inserted into the antebrachial fascia and the medial epicondyle of the humerus (7).

Other unusual sites of CBM insertion are the tendon of the latissimus dorsi (minor coracobrachial muscle of Cruveilhier), the skin and fascia of the axilla, the tendon of the subscapular muscle $(4,15)$, the surgical neck of the humerus, the intertubercular sulcus (3), the medial head of the triceps (5), the tendon of the teres major (9), and the olecranon process (10). As regards the embryological explanation of such varieties, it should be emphasized that the upper limbs' muscles are derived from the lateral meso- derm. In order for a certain muscle to be formed, muscle primordia are fused. Some muscle primordia disappear through cell death. The persistence of some cells between the CBM and the biceps brachialis muscle may result in an accessory CBM belly (1, 14). Alternatively, someone could hypothesize that during the CBM's embryological development, a combination occurs of the CBM's tangential splitting into two heads and migration of the CBM's superficial head into more distal and medial region (16). As regards the CBM's action, the muscle flexes the arm forward and medially, especially from a position of brachial extension (8). The CBM, according to Wood, resembles the triceps adductor femoris. In particular, the short upper portion of the muscle corresponds to the adductor brevis, the middle portion to the adductor longus and the long lower portion of the CBM to the adductor magnus. In accordance with Wood's suggestions, the CBM's middle and lower portions act as the adductor and elevator of the upper arm. In addition, the lower portion will render the brachial fascia tense. The short upper portion of the CBM acts as an external rotator of the humerus. Moreover, in cases of the CBM's insertion into the shoulder joint capsule, Wood considered that this portion draws the capsule forward, preventing it being rucked up into folds, or pinched in extreme adduction (9). Ilayperuma et al. considered that the CBM acts as an enhancing muscle for the tendon of the short head of the biceps brachii, since it takes its origin from both sides of that tendon. These authors claimed that CBM provides the optimum position for the aforementioned tendon, to exert the proper action of the muscle on the glenohumeral joint (17).

As regards the potential functional significance of our finding, we consider that the additional superficial CBM head could potentially induce symptoms of median or ulnar neuropathy in instances of its hyper- 
trophy or traumatic injury. Gessini et al. mentioned that the hypertrophic coracobrachialis longus muscle may be an etiological factor for potential entrapment of the median nerve and/or vascular disturbances, due to compression of the brachial artery (18). Ray et al. rightly asserted that, due to the fact that it is difficult to distinguish median or ulnar neuropathy in the upper arm from that in the lower arm, these types of CBM variation should be kept in mind (6). Furthermore, awareness of the CBM's variations should be highlighted for surgeons in that field, since that muscle may be utilized as a guide for the location of the axillary artery, as a vascularized graft for treatment of long standing facial palsy (2), for post-mastectomy reconstruction, and in defects of the axillary and infraclavicular regions (1).

\section{Conclusion}

A profound understanding of this muscular variation is indispensable for physicians and surgeons in that field for interpretation of sensory, motor and vascular disturbances of the upper limb, as well during preparation of the CBM to be utilized as a free or vascularized graft for reconstruction of defects of adjacent areas, or for long standing facial palsy.

\section{What is already known on this topic}

The origin of the coracobrachialis muscle (CBM) as well as its insertion display great variability. As regards CBM's insertion, which is the case in the current study, it may be located in various anatomical sites, from the shoulder joint capsule to the medial epicondyle, the olecranon process or the antebrachial fascia. Specifically, this muscle may insert into variable anatomical sites, such as the surgical neck of the humerus, the tendon of the latissimus dorsi, the medial head of the triceps, the brachial fascia, the medial epicondyle of the humerus, or the antebrachial fascia.

\section{What this study adds}

Some cases of additional CBM heads have been reported in the relevant literature. However, the presence of a bicipital $C B M$ with abnormal insertion of its superficial head, as in the current study, where its superficial portion participates in the formation of a musculo-aponeurotic tunnel including the me- dian, ulnar and medial antebrachial cutaneous nerves, as well the brachial vessels, is not so commonly detected. As regards the potential functional significance of our finding, we consider that the additional superficial CBM head could potentially induce symptoms of median or ulnar or medial antebrachial cutaneous neuropathy, or vascular disorders in instances of its hypertrophy, hematoma or strong CBM contraction under resistance.

Authors' contributions: Conception and design: GP and KK; Acquisition, analysis and interpretation of data: GP, KI and TB; Drafting the article: GP, KK and PK; Revising it critically for important intellectual content: GP and KK; Approved final version of the manuscript: GP, KK, KI, TB and PK.

Conflict of interest: The authors declare that they have no conflict of interest.

\section{References}

1. Grim M. Ultrastructure of the ulnar portion of the contrahent muscle layer in the embryonic human hand. Folia Morphol (Praha). 1972;20(2):113-5.

2. Taylor GI, Cichowitz A, Ang SG, Seneviratne $\mathrm{S}$, Ashton H. Comparative anatomical study of gracilis and coracobrachialis muscle implication for facial animation. Plast Reconstr Surg. 2003;112(1):20-30.

3. Le Double AF. Traite des variation du systeme musculaire de I'homme. Tome 2. Paris: Schleicher Frères; 1897. p. 21-32.

4. Bergman RA, Thompson SA, Afifi AK, Saadeh FA. Compendium of Human Anatomic Variation. Baltimore: Urban and Schwarzenberg; 1988. p. 10-1.

5. El-Naggar MM, Zahir Fl. Two bellies of the coracobrachialis muscle associated with a third head of the biceps brachii muscle. Clin Anat. 2001;14(5):379-82.

6. Ray B, Rai AL, Roy TS. Unusual insertion of the coracobrachialis muscle to the brachial fascia associated with high division of brachial artery. Clin Anat. 2004;17(8):672-6.

7. Kopuz C, Içten N, Yildirim M. A rare accessory coracobrachialis muscle: a review of the literature. Surg Radiol Anat. 2003;24(6):406-10.

8. Williams PL, Bannister LH, Berry MM, Collins P, Dyson M, Dussek JE, et al., editors. Gray's Anatomy. 38th ed. Edinburgh: Churchill Livingstone; 1995. p. 842.

9. Wood J. On human muscular variations and their relation to comparative anatomy. J Anat Physiol. 1867;1(1):44-59. 
10. Vollala VR, Nagabhooshana S, Bhat SM, Potu BK, Rakesh V. Multiple accessory structures in the upper limb of a single cadaver. Singapore Med J. 2008;49(9):e254-8.

11. Mori M. Statistics on the musculature of the Japanese. Okajimas Folia Anat Jpn. 1964;40:195-300.

12. Paraskevas G, Natsis K, Ioannidis O, Papaziogas B, Kitsoulis P, Spanidou S. Accessory muscles in the lower part of the anterior compartment of the arm that may entrap neurovascular elements. Clin Anat. 2008;21(3):246-51.

13. Chouke KS. Variation of the coracobrachialis muscle. Anat Rec. 1924;27(3):157-61.

14. Gupta G, Singh K, Chhabra S, Srivastava SK, Gupta V. Accessory coracobrachialis: a case report with its morphological and clinical significance. Surg Radiol Anat. 2012;34(7):655-9.
15. Macalister A. Additional observations on muscular anomalies in human anatomy. Third series with a catalogue of the principal muscular variations hitherto published. Trans Roy Irish Acad. 1875;25:1-130.

16. Arey LB. Developmental Anatomy. A Textbook and Laboratory Manual of Embryology. 6th ed. Philadelphia: W.B. Saunders Company; 1960. p. 430-1.

17. Ilayperuma I, Nanayakkara BG, Hasan R, Uluwitiya SM, Palahepitiya KN. Coracobrachialis muscle: morphology, morphometry and gender differences. Surg Radiol Anat. 2016;38(3):335-40.

18. Gessini L, Jandolo B, Pietrangeli A. Entrapment neuropathies of the median nerve at and above the elbow. Surg Neurol. 1983;19(2):112-6. 